\title{
Constant-Rate Oblivious Transfer from Noisy Channels
}

\author{
Yuval Ishai $^{1, \star}$, Eyal Kushilevitz ${ }^{1, \star \star}$, Rafail Ostrovsky ${ }^{2, \star \star \star}$ \\ Manoj Prabhakaran ${ }^{3, \dagger}$, Amit Sahai ${ }^{2, \ddagger}$, and Jürg Wullschleger ${ }^{4, \S}$ \\ 1 Technion, Haifa, Israel \\ \{yuvali, eyalk\}@cs.technion.il \\ 2 University of California, Los Angeles \\ \{rafail, sahai\}@cs.ucla.edu \\ 3 University of Illinois, Urbana-Champaign \\ mmp@cs . uiuc.edu \\ 4 Université of Montréal and McGill University \\ juerg@wulli.com
}

\begin{abstract}
A binary symmetric channel (BSC) is a noisy communication channel that flips each bit independently with some fixed error probability $0<p<1 / 2$. Crépeau and Kilian (FOCS 1988) showed that oblivious transfer, and hence general secure two-party computation, can be unconditionally realized by communicating over a BSC. There has been a long line of works on improving the efficiency and generality of this construction. However, all known constructions that achieve security against malicious parties require the parties to communicate poly $(k)$ bits over the channel for each instance of oblivious transfer (more precisely, $\left(\begin{array}{l}2 \\ 1\end{array}\right)$ bit-OT) being realized, where $k$ is a statistical security parameter. The question of achieving a constant (positive) rate was left open, even in the easier case of realizing a single oblivious transfer of a long string.

We settle this question in the affirmative by showing how to realize $n$ independent instances of oblivious transfer, with statistical error that vanishes with $n$, by communicating just $O(n)$ bits over a BSC. As a corollary, any boolean circuit of size $s$ can be securely evaluated by two parties with $O(s)+\operatorname{pol} y(k)$ bits of communication over a BSC, improving over the $O(s) \cdot \operatorname{poly}(k)$ complexity of previous constructions.
\end{abstract}

* Work done in part while visiting UCLA. Supported by ERC Starting Grant 259426, ISF grant 1361/10, and BSF grant 2008411.

** Work done in part while visiting UCLA. Supported by ISF grant 1361/10 and BSF grant 2008411.

$\star \star \star$ Research supported in part by DARPA, IBM Faculty Award, Xerox Innovation Group Award, the Okawa Foundation Award, Intel, Teradata, NSF grants 0830803, 0916574, BSF grant 2008411 and U.C. MICRO grant.

† Supported by NSF grant CNS 07-47027.

¥ Research supported in part from a DARPA/ONR PROCEED award, NSF grants 0916574 and 0830803, a Xerox Foundation Award, a Google Faculty Research Award, an equipment grant from Intel, and an Okawa Foundation Research Grant.

$\S$ Research supported by the Canada-France NSERC-ANR project FREQUENCY.

P. Rogaway (Ed.): CRYPTO 2011, LNCS 6841, pp. 667-684, 2011.

(C) International Association for Cryptologic Research 2011 


\section{Introduction}

One of the attractive features of modern cryptography is its ability to "turn lemons into lemonade." Indeed, traditional complexity-based cryptography turns computational intractability, a major obstacle tackled by Computer Science, into a blessing. The present work is concerned with a similar phenomenon in the context of information-theoretic cryptography: the ability to turn noise, a major obstacle tackled by Information Theory, into a blessing.

Originating from the seminal work of Wyner [38] on the usefulness of noise for secure communication, there has been a large body of work on basing various cryptographic primitives on different types of noisy communication channels. The most fundamental type of a noisy channel in information theory is the binary symmetric channel (BSC). A BSC with crossover probability $p$, where $0<p<\frac{1}{2}$, flips each communicated bit independently with probability $p$.

In 1988, Crépeau and Kilian [10] showed that two parties can make use of a BSC to realize oblivious transfer (OT) 3215] with unconditional security. By OT we refer by default to $\left(\begin{array}{l}2 \\ 1\end{array}\right)$-bit-OT, a protocol which allows a receiver to select exactly one of two bits held by a sender without revealing the identity of the received bit to the sender. We require by default that security hold even against malicious parties. It is known that OT on a pair of $m$-bit strings reduces to $O(m)$ instances of bit-OT [4]. Much more broadly, OT can be used as a basis for general secure two-party computation 39192624. This settles the main feasibility question concerning the cryptographic power of a BSC.

In contrast to the basic feasibility question, the corresponding efficiency questions are far less understood. To explain the main relevant issues, it is instructive to draw an analogy with classical information theory. A naive approach to send $n$ bits of information over a noisy channel is to do it bit-wise, by repeating every bit $k$ times. A major breakthrough in information theory was the seminal result of Shannon 33 that by sending bits in blocks and by using the right encoding, one can achieve a constant transmission rate, namely use only a constant number of channel transmissions per information bit with error that vanishes with $n$. One can analogously define the notion of a constant-rate protocol for $\mathrm{OT}$ from BSC (or a constant-rate reduction of OT to BSC) as a protocol which realizes $n$ independent instances of OT with negligible (in $n$ ) statistical error 1 by exchanging $O(n)$ bits over the channel 2 In such a protocol, the amortized communication complexity for each instance of OT tends to a constant which is independent of the desired level of security.

The existence of a constant-rate protocol for OT from BSC has been a longstanding open question. The original protocol from [10] required $O\left(k^{11}\right)$ bits of communication over a BSC to realize each instance of OT with error $2^{-k}$. This communication overhead was subsequently improved by Crépeau [9] to $O\left(k^{3}\right)$.

\footnotetext{
${ }^{1}$ By the error of OT or other secure computation protocol we refer to the statistical simulation error under standard simulation-based definitions 5/6 18.

2 This is the best one can hope for up to the exact constant. Indeed, it is known that $\Omega(n)$ bits over the BSC are necessary even if one additionally allows unlimited communication over a noiseless channel 35.
} 
A major progress was made by Harnik et al. 20, who showed that constant rate can be achieved in the semi-honest model, in which parties do not deviate from the protocol except for trying to infer additional information from their view.

Constant-rate protocols for string OT, realizing a single selection between two $n$-bit strings by communicating $O(n)$ bits over the channel, are considerably easier to obtain. (Indeed, known reductions [4] can be used to get constant-rate string-OT from constant-rate bit-OT, but not the other way around.) Constantrate string-OT protocols from an erasure channel, which erases every bit with probability $0<p<1$ and informs the receiver of the erasures, were presented in $30 \mid 22$.

To summarize the prior state of the art, constant-rate protocols for bit-OT from BSC were only known in the semi-honest model, and constant-rate stringOT protocols could only be based on an erasure channel. The existence of constant-rate bit-OT protocols from a BSC (or even from an erasure channel) as well as the existence of constant-rate string-OT protocols from a BSC were left open.

\section{$1.1 \quad$ Our Results}

We settle the above questions in the affirmative by presenting a statistically secure protocol which realizes $n$ independent instances of OT, with $2^{-k}$ error, in which the parties communicate only $O(n)+\operatorname{poly}(k)$ bits over a BSC 3 This should be compared to the $n \cdot \operatorname{poly}(k)$ bits required by previous constructions.

Combining the above main result with known results for secure two-party computation based on OT 24] we get the following corollaries:

- Any boolean circuit of size $s$ can be securely evaluated by two parties with $O(s)+p o l y(k)$ bits of communication over a BSC, improving over the $O(s)$. poly $(k)$ complexity of previous constructions.

- Applying the previous corollary, any discrete memoryless channel (described by rational crossover probabilities) can be faithfully emulated by a BSC at a constant rate.

Our techniques can be used to get similar results based on any "non-trivial" channel rather than just a BSC. We defer this generalization to the full version of this paper.

\subsection{Overview of Techniques}

Our construction uses a novel combination of previous results on OT from BSC 1020 , recent techniques from the area of secure computation 824, and some new tools that may be of independent interest.

\footnotetext{
${ }^{3}$ The protocol also involves a similar amount of communication over a noiseless channel. This additional communication can be implemented using the BSC with a constant rate.
} 
Among the new general-interest tools is a so-called "Statistical-to-Perfect Lemma," showing roughly the following. Given a 2-party functionality $\mathcal{F}_{f}$ for securely evaluating a function $f$ and $0 \leq \delta \leq 1$, we define $\widetilde{\mathcal{F}}_{f}^{(\delta)}$ to be an " $\delta$ faulty" version of $\mathcal{F}_{f}$ that with probability $\delta$ allows the adversary to learn the inputs and have full control over the outputs but otherwise behaves normally. The lemma says that any $\epsilon$-secure protocol for $\mathcal{F}$ in a $\mathcal{G}$-hybrid model (i.e., using oracle access to $\mathcal{G}$ ) perfectly realizes the functionality $\widetilde{\mathcal{F}}_{f}^{(\delta)}$ in the $\mathcal{G}$-hybrid model, where $\delta$ tends to 0 with $\epsilon$ (but inherently grows with the size of the input domain). The above lemma allows one to take an arbitrary (and possibly inefficient) protocol for OT from a noisy channel, such as the one from [10, and use it with a sufficiently large security parameter to get a perfectly secure implementation of $\widetilde{\mathcal{F}}_{\mathrm{OT}}^{(\delta)}$, for an arbitrarily small constant $\delta>0$, while communicating just a constant number of bits (depending on $\delta$ ) over the channel.

This calls for the use of OT combiners [2120]31, which combine $n$ OT implementation candidates of which some small fraction may be faulty into $m<n$ good instances of OT. A similar high level approach was used in [20] to solve our main question in the semi-honest model. While in the semi-honest model there are constant-rate combiners (tolerating a constant fraction of faulty candidates with $m=\Omega(n)$ ) that make only a single use of each OT candidate [20, known constant-rate OT combiners in the malicious model require a large number of calls to each candidate, making them insufficient for our purposes. Instead, we take the following alternative approach.

1. We give a direct construction of a constant-rate protocol for string-OT from a BSC. (As discussed above, such a result was only known for the easier cases of an erasure channel or in the semi-honest model.) The protocol employs previous protocols for OT from BSC [10, the completeness of OT for secure two-party computation [26 24], techniques from secure multiparty computation (including the use of algebraic-geometric multiplicative secret sharing 8 25]), and privacy amplification techniques 32. Its analysis relies on the Statistical-to-Perfect lemma discussed above.

2. We extend the IPS protocol compiler 24 to apply also when the so-called "inner protocol" can employ a BSC channel. The main difficulty is that even when being forced to reveal their secrets, parties can use the uncertainty of the channel to lie without taking the risk of being caught. We address this difficulty in a natural way by employing statistical tests to ensure that significant deviations are being caught with high probability. The extended protocol compiler requires the inner protocol to satisfy an intermediate notion of security, referred to as "error-tolerance," that is stronger than security in the semi-honest model and weaker than security in the malicious model.

3. We instantiate the ingredients required by the extended compiler from Step 2 as follows. The so-called "watchlists" are implemented using string-OTs obtained via the protocol described in Step 1 above. The outer protocol is an efficient honest-majority MPC protocol for $n$ instances of OT (see 23], building on [138]). The error-tolerant inner protocol is based on an error-tolerant constant-rate OT combiner from [20]. 


\subsection{Related Work}

There is a very large body of related work on cryptography from noisy channels that was not accounted for in the above survey, and even here we can only give a very partial account. For the question of basing other cryptographic primitives (such as key agreement and commitment) on noisy channels see [2 32814 36 37] and references therein. The question of characterizing the types of channels on which OT can be based was studied in 27111412 37]. A general approach for converting feasibility results for OT from noisy channels into constant-rate protocols in the semi-honest model was given in 25. Our work introduces a similar conversion technique that can be applied in the malicious model.

\section{Preliminaries}

Some of our results and analysis (in particular Theorem 11) apply to general 2-party secure function evaluation (SFE) functionalities. Such a functionality is characterized by a pair of functions $f=\left(f_{A}, f_{B}\right), f_{A}: \mathcal{X} \times \mathcal{Y} \rightarrow \mathcal{Z}_{A}$ and $f_{B}: \mathcal{X} \times \mathcal{Y} \rightarrow \mathcal{Z}_{B}$ for (often finite) domains $\mathcal{X}, \mathcal{Y}$ and ranges $\mathcal{Z}_{A}, \mathcal{Z}_{B}$. We will refer to such an $f$ as a 2-pary function. We associate a functionality $\mathcal{F}_{f}$ with a 2-party function $f$, which behaves as follows: $\mathcal{F}_{f}$ waits for inputs from both parties, and computes the respective outputs. Then if either party is corrupted, it sends the corresponding output to that party. Then it waits for an instruction from the adversary to release the output(s) to the uncorrupted party (or parties). We shall refer to such a functionality $\mathcal{F}_{f}$ as a 2-party SFE functionality.

The two main functionalities in this work are $\mathcal{F}_{\text {BSC }}$ and $\mathcal{F}_{\text {OT }}$. The $\mathcal{F}_{\text {BSC func- }}$ tionality (BSC stands for Binary Symmetric Channel) takes as input a bit $x$ from one of the parties (Alice), and outputs a single bit $z$ to the other party (Bob) such that $\operatorname{Pr}[x \neq z]=p$ for some fixed constant probability strictly less than half. (Note that this is a randomized functionality.) $\mathcal{F}_{\text {OT }}$ is an SFE functionality, associated with a function defined by $f_{B}\left(x_{0}, x_{1} ; b\right)=x_{b}$ where $x_{0}, x_{1}, b$ are single bits each; for $\mathcal{F}_{\text {OT }} f_{A}$ is a constant function. The functionality $\mathcal{F}_{\text {string-OT }}$ is similar to $\mathcal{F}_{\mathrm{OT}}$, but the inputs from Alice $x_{0}, x_{1}$ are longer strings.

For every 2-party SFE functionality $\mathcal{F}_{f}$, we define a weakened variant $\tilde{\mathcal{F}}_{f}^{(p)}$ where $0 \leq p \leq 1$ is a constant error probability in the following sense. When invoked, an instance of $\widetilde{\mathcal{F}}_{f}^{(p)}$ would first generate a random bit which is 1 with probability $p$. Note that the bit is sampled before receiving inputs from any party. If the bit is 0 , then the functionality behaves exactly as $\mathcal{F}_{f}$. Otherwise, if the bit is 1 , then the functionality yields itself to adversarial control: i.e., the input(s) it receives are passed on to the adversary, and the adversary specifies the outputs to be sent (and when they should be sent) to the honest party (parties). In this case, even if neither party interacting with the functionality is corrupt, the functionality will allow the adversary to control it.

The main security definition we use is of statistical Universally Composable (UC) security [6]. The level of security - called statistical error - is indicated 
by the maximum distinguishing advantage between the real execution of the protocol and a simulated execution involving the ideal functionality that any environment can get (the distinguishing advantage being the difference in probabilities of the environment outputting 1 when interacting with the two systems). We require that the statistical error goes down as $2^{-\Omega(k)}$, where $k$ is the security parameter. The computational complexity of the protocols should be polynomial in $k$ and the input size. For intermediate constructions (and in Theorem 10) we consider perfect security as well.

We say that a protocol $\Pi$ is in the $\mathcal{G}$-hybrid model if the parties can initiate and interact with (any number of) instances of the ideal functionality $\mathcal{G}$. Our goal is to give a "constant-rate" protocol for $\mathcal{F}_{\mathrm{OT}}$ in the $\mathcal{F}_{\mathrm{BSC}}$-hybrid model. A protocol $\Pi$ in the $\mathcal{G}$-hybrid model is said to be a constant-rate protocol for a functionality $\mathcal{F}$, if the total communication in $\Pi$ (including communication with instances of $\mathcal{G}$ ) is $O(\ell)+\operatorname{poly}(k)$, where $\ell$ is the total communication with $\mathcal{F}$. We will be interested in realizing parallel instances of a target functionality, given the number of instances as a parameter (during run-time). More formally we can define a functionality $\mathcal{F}^{*}$ which takes $\ell$ as an initial input from one of the parties, and then implements $\ell$ parallel copies of $\mathcal{F}$. Note that when $\mathcal{F}$ and $\mathcal{G}$ are finite functionalities (i.e., with the total communication with a single instance upperbounded by a constant, as is the case for $\mathcal{F}_{\text {OT }}$ and $\mathcal{F}_{\text {BSC }}$ ), to securely realize $\mathcal{F}^{*}$, a constant-rate protocol $\Pi$ will instantiate only $O(\ell)+\operatorname{poly}(k)$ instances of $\mathcal{G}$. (For simplicity, we shall refer to $\Pi$ as a protocol for $\mathcal{F}$, rather than $\mathcal{F}^{*}$.)

An Arithmetic Encoding Scheme. Our protocol (particularly, the sub-protocol in Section 4.1) relies on an efficient secret-sharing scheme that supports entrywise addition and multiplication of shared vectors. Following the terminology of [7], we refer to such a scheme as an arithmetic encoding scheme. Our abstraction captures the useful features of algebraic-geometric secret-sharing, introduced in [8] (see 2517] for related abstractions).

Our notion of arithmetic encoding is parameterized by a tuple $\left(\mathbb{F}, \rho, \delta, \delta^{\prime}\right)$ and is defined by three efficient algorithms (Encode, Encode', Decode ${ }^{\prime}$ ). Here $\mathbb{F}$ is a constant-size finite field, $\rho, \delta, \delta^{\prime}$ are positive constants less than 1 , and the three algorithms satisfy the following properties.

- Encode and Encode' define constant-rate, probabilistic encodings of vectors over $\mathbb{F}$. More precisely, for every integer $m>0$, there is an $n$, with $m>$ $\rho n$, such that Encode and Encode' probabilistically map vectors in $\mathbb{F}^{m}$ to $\mathbb{F}^{n}$. Further, Encode and Encode' are linear: i.e., each entry of Encode $(x)$ (respectively, Encode $(x)$ ) is a linear function of the entries of $x$ and a set of independent random elements.

- The joint distribution of any $\lfloor\delta n\rfloor$ entries of the output of Encode $(x)$ is independent of the input $x$.

- Decode ${ }^{\prime}$ is an efficient $\delta$-error-correcting decoder for Encode'. More precisely, we require that if $y$ has Hamming distance at most $\delta n$ from a vector in the support of $\operatorname{Encode}^{\prime}(x)$, then $\operatorname{Decode}^{\prime}(y)=x$. 
- We require the following "homomorphic" properties. For any $X, Y, X^{\prime}, Y^{\prime}$ in the support of $\operatorname{Encode}(x), \operatorname{Encode}(y), \operatorname{Encode}^{\prime}\left(x^{\prime}\right), \operatorname{Encode}^{\prime}\left(y^{\prime}\right)$, respectively:

- $X * Y$ is in the support of $\operatorname{Encode}^{\prime}(x * y)$

- $X^{\prime}+Y^{\prime}$ is in the support of Encode' $\left(x^{\prime}+y^{\prime}\right)$

where $*$ and + denote entrywise multiplication and addition over $\mathbb{F}$ respectively.

- We require Encode' to be sufficiently randomizing. Note that $\operatorname{Encode}^{\prime}(x)$ is uniform over an affine subspace of $\mathbb{F}^{n}$ whose dimension is at most $n-m$. We require that this dimension be at least $n-m\left(1+\delta^{\prime}\right)$.

An arithmetic encoding scheme with the above properties can be obtained from the classes of algebraic geometric codes used in [8]. See Appendix $\mathrm{A}$ for details.

\section{Statistical Security to Perfect Security}

A crucial ingredient in our constructions and analysis is the ability to consider a weakly secure protocol to be a perfectly secure protocol for a weaker variant of the functionality. More precisely, we show the following.

Theorem 1. Let $f: \mathcal{X} \times Y \rightarrow \mathcal{Z}_{A} \times \mathcal{Z}_{B}$ be a 2-party function, and $\mathcal{F}_{f}$ the secure function evaluation functionality for $f$. Suppose $\mathcal{G}$ is a 2-party functionality and $\Pi$ is a D-round protocol such that $\Pi U C$ securely realizes $\mathcal{F}_{f}$ in the $\mathcal{G}$-hybrid model, with a statistical security error of $\epsilon$. Then $\Pi$ UC securely realizes $\widetilde{\mathcal{F}}_{f}^{(p)}$ in the $\mathcal{G}$-hybrid model with perfect security, where $p=D|\mathcal{X}||\mathcal{Y}| \epsilon$.

Above, if $\Pi$ is only standalone-secure for $\mathcal{F}_{f}$, then the same conclusion holds for standalone security of $\Pi$ for $\widetilde{\mathcal{F}}_{f}^{(p)}$.

This result gives a powerful composition theorem when multiple instances of the protocol $\Pi$ are used together. Note that by UC security, it is indeed the case that if $k$ copies of $\Pi$ are run, one could instead consider $k$ copies of $\mathcal{F}$, with a statistical security error bounded by $k \epsilon$. However, if $\epsilon$ is not negligible, say $\epsilon>1 / k$, then this bound gives us no useful security guarantee. What the above result does is to give a strong security guarantee for the case when $\epsilon$ is non-negligible, or even when it is a constant. It says that when $k$ copies of $\Pi$ are run, it roughly yields $(1-p) k$ copies of $\mathcal{F}$ (mixed with about $p k$ copies under adversarial control). In fact, it is further guaranteed that which copies will be corrupted is not under adversarial control.

In the full version we show that it is unavoidable that $p$ is bigger than $\epsilon$ by a factor that grows linearly with the domain size of the function.

We give a high-level idea of how we prove the above theorem. Given the systems corresponding to REAL and IDEAL executions, the overall approach is to decompose each of the REAL and IDEAL systems into two parts - REAL , REAL $_{1}$ and IDEAL ${ }_{0}, \mathrm{IDEAL}_{1}$ - so that REAL $\mathrm{L}_{0}$ and IDEAL $\mathrm{I}_{0}$ are identical and carry much of the "mass" of the systems; then we construct a new ideal system by combining $\mathrm{IDEAL}_{0}$ and REAL ${ }_{1}$, to get a system that is identical to the REAL system. Here, a combination of two systems means that with a fixed probability one of the two 
systems is chosen (corresponding to whether $\widetilde{\mathcal{F}}_{f}^{(p)}$ lets the adversary control it or not, corresponding to choosing IDEAL 1 and $\mathrm{IDEAL}_{0}$ respectively): in particular, the simulator in the new system is not allowed to influence this choice. Further and this is the main difficulty in the proof - we need to ensure that IDEAL $_{0}$ can be implemented by a simulator interacting with $\mathcal{F}_{f}$ (without access to an honest party's inputs or outputs); to implement $\mathrm{REAL}_{1}$ the simulator may control the functionality as well.

Note that Theorem 1 is related to Lemma 5 in [29]. The main difference are the abovementioned restrictions on the system $\mathrm{IDEAL}_{0}$ which require extra care in our proof.

Splitting the systems in this manner needs to be carefully defined, see full version for details. Here we illustrate this by a toy example, to give a sense of how the simulator for perfect security is derived from the simulator for statistical security. The protocol we consider is for a degenerate 2-party function $f$ which provides a constant output to both parties. Further, it takes a fixed input from Alice $(|\mathcal{X}|=1)$ and takes a bit from $\operatorname{Bob}(\mathcal{Y}=\{0,1\})$. The protocol $\Pi$ for our example consists of a single message $z$ from Bob to Alice, which is equal to $y$ with probability $\frac{1}{2}$ and $\perp$ otherwise. We shall consider the case when Alice is corrupt and Bob is honest. Further we need to consider only a "dummy adversary" who simply allows the environment to play the role of Alice in the (real) protocol. The simulator simulates a message from Bob, which is equal to $\perp$ with probability $\frac{1}{2}$, and a uniformly chosen bit otherwise. It is easy to see that this simulation is good up to a statistical distance of $\frac{1}{4}$.
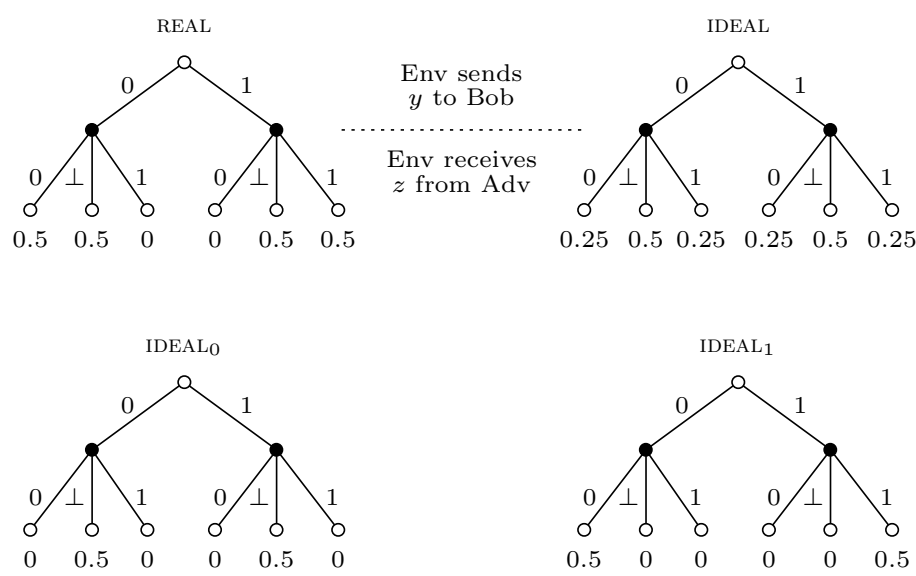

Fig. 1. An example to illustrate Theorem 1. The protocol used in the example (in which Bob sends a single message to Alice - please see text) is depicted as the interaction of a system REAL with the environment. The original simulated system is IDEAL. The modified simulation (for a functionality that yields to the adversary with probability $0.5)$ is obtained as the combination IDEAL $0+$ IDEAL $_{1}$ which is exactly the same as the REAL system. 
In Figure 1 we illustrate this example using what we call interaction trees, which capture an execution involving a system (REAL or IDEAL) and an environment. The edges from the top node in these trees correspond to the two possible inputs that the environment can give to Bob $(y=0$ and $y=1)$. The edges out of the black nodes correspond to corrupt Alice reporting the (only) message it receives from Bob in the protocol: this can be one of 0 , 1 , or $\perp$. The leaves correspond to complete transcripts. The probabilities of the system reaching a leaf, provided the environment sends the messages (in our case, just $y$ ) that lead to that leaf is considered the "weight" assigned to that leaf by the system.

The top-left figure corresponds to the real execution of the protocol, and the top-right corresponds to the ideal execution. Note that in the simulation, the behavior of the simulator is independent of the input $y$. Then we obtain a "partial system" (with total weight only 0.5 , for each value of $y$ ), IDEAL $\mathrm{I}_{0}$ by comparing the REAL and IDEAL systems. In this example, IDEAL $\mathrm{I}_{0}$ is obtained by retaining in each leaf the minimum of the weights assigned by the two systems, on that leaf, but for any choice of $y$. We will use the ideal functionality $\widetilde{\mathcal{F}}_{f}^{(p)}$, with $p=\frac{1}{2}$, since that is the weight not retained by $\operatorname{IDEAL}_{0}$.

IDEAL $_{1}$ is obtained by "subtracting" IDEAL ${ }_{0}$ from REAL, so that the combination of $I_{D E A L}$ and IDEAL 1 is indeed REAL. In doing this we needed to ensure that weights induced by $\mathrm{IDEAL}_{0}$ are no more than what REAL assigns (so that the system REAL - IDEAL 0 does not have negative weights). Also we needed to ensure that $\operatorname{IDEAL}_{0}$ can be implemented by a simulator which does not have access to $y$. Note that to implement IDEAL $_{1}$, the simulator will need to know $y$.

In going from this toy example to the general case poses several issues. Here the simulator for $\mathrm{IDEAL}_{0}$ was determined without considering the interaction between the simulator and the functionality. (Indeed, there was little interaction between the two.) In general we cannot afford to do this. To properly take into account how the simulator's behavior depends on what it learns from the functionality, we consider a separate interaction which the simulator is the system and it interacts with an "enhanced environment" consisting of the original environment and the functionality. But the original statistical security guarantee is only against normal environments (and indeed, does not make sense against enhanced environments, since in the real execution there is no ideal functionality present). This requires us to relate the behavior of the enhanced environment to the behavior of the environment in the ideal world.

The final proof uses several carefully defined quantities for the three systems (the real and ideal executions, and the simulator system), and shows how one can define IDEAL $\mathrm{I}_{0}$ which can be implemented without using $y$, ensures that it can be extended to a perfect simulation (i.e., that the remainder of the simulation is a non-negative system), while retaining as much weight as possible (to keep $p$ low as promised in Theorem 10. 


\section{A Constant-Rate OT Protocol}

In this section we present our constant-rate protocol for $\mathcal{F}_{\text {OT }}$ in the $\mathcal{F}_{\text {BSC-hybrid }}$ model. The construction follows the paradigm of the IPS compiler [24] of combining an outer protocol secure in the honest-majority setting, with an inner protocol secure in the passive corruption (semi-honest) setting, using "watchlists" implemented using string-OTs. For this we need to instantiate these components in the $\mathcal{F}_{\mathrm{BSC}}$-hybrid model, and also extend the IPS compiler so that it admits an inner protocol in the $\mathcal{F}_{\mathrm{BSC}}$-hybrid model. We outline these steps below, and present the details in the subsequent sections.

- In order to construct the inner protocol, we will need a constant-rate OT protocol using $\mathcal{F}_{\mathrm{BSC}}$, that is secure against adaptive passive corruption. However, since monitoring the use of a $\mathcal{F}_{\mathrm{BSC}}$ functionality (which inherently allows errors) is harder than monitoring the use of the $\mathcal{F}_{\text {OT }}$ functionality we will need a somewhat stronger security guarantee from this protocol (namely, passive security should hold even when a small constant fraction of the $\mathcal{F}_{\text {BSC }}$ instances can be corrupted). We shall formalize this notion of "error-tolerance" and observe that a protocol in [20] already has the requisite properties (Lemma 2).

- The next step is to construct a constant-rate string-OT protocol from $\mathcal{F}_{\mathrm{BSC}}$, with security against active corruption (Lemma 1). The protocol implements a single instance of string-OT (i.e., takes only one choice bit as input from the receiver), and the rate refers to the ratio of the length of the string to the number of instances of $\mathcal{F}_{\mathrm{BSC}}$ used. This crucially relies on Theorem 1 which states that a weakly secure protocol for a functionality is a perfectly secure protocol for a weak version of the same functionality.

- The final step involves an extension of the IPS compiler 24] wherein the "inner-protocol" is in the $\mathcal{F}_{\text {BSC }}$-hybrid model (rather than in the $\mathcal{F}_{\text {OT }}$-hybrid model) and enjoys error-tolerance (Lemma 3).

The extended IPS compiler from above is used to combine an appropriate constant-rate 4 outer protocol for $\mathcal{F}_{\text {OT }}$ (based on [138], as used in [24]) with an error-tolerant inner protocol obtained from the first step, using watchlists implemented using string-OTs from the second step.

To implement $n$ instances of $\mathcal{F}_{\text {OT }}$, the resulting compiled protocol will invoke the string-OT protocols $O(k)$ times with $O(n / k)$ long strings. Since these stringOTs are implemented using the constant-rate protocol from the second step, the compiled protocol uses a total of $O(n)$ instances of $\mathcal{F}_{\mathrm{BSC}}$ for the watchlists.

Similarly the compiled protocol invokes $k$ instances of the inner-protocol (for a functionality defined by the outer protocol). Originally, each instance

\footnotetext{
${ }^{4}$ Here the constant-rate refers to the total communication in the protocol, and the total computation of all the servers per instance of $\mathcal{F}_{\text {Oт }}$ produced. More precisely, regarding the computational complexity of the servers, we are interested in the complexity of a passive-secure protocol for implementing the server computations, and it is only the so-called "type II" computations of the servers that contribute to this. See 24 for details.
} 
of this inner-protocol can be implemented using $O(n / k)$ instances of $\mathcal{F}_{\text {OT }}$, and is passive-secure in the $\mathcal{F}_{\text {OT-hybrid model. We shall replace the } \mathcal{F}_{\text {OT }} \text { instances }}$ used by the inner protocol with the constant-rate error-tolerant protocol from the first step. This results in an error-tolerant inner protocol in the $\mathcal{F}_{\text {BSC-hybrid }}$ model (for the same functionality as the original inner-protocol), which uses $O(n / k)$ instances of $\mathcal{F}_{\mathrm{BSC}}$. Thus overall, for the inner-protocol instances too, the compiled protocol uses $O(n)$ instances of $\mathcal{F}_{\mathrm{BSC}}$.

In the following sub-sections we describe how the above three steps are carried out, and what precise security guarantees they provide. Then, by following the above sketched construction we obtain our main result.

Theorem 2. There exists a UC-secure constant-rate protocol for $\mathcal{F}_{\mathrm{OT}}$ in the $\mathcal{F}_{\mathrm{BSC}}$-hybrid model. That is, there is a protocol that securely realizes $n$ parallel, independent instances of $\mathcal{F}_{\mathrm{OT}}$ with statistical error $2^{-k}$, with $O(n)+$ poly $(k)$ bits of communication (including communication over $\mathcal{F}_{\mathrm{BSC}}$ ).

An important corollary of implementing oblivious transfer is that it can be used to implement arbitrary function evaluation, quite efficiently [24]. Thus combined with the main result of [24] we have the following.

Corollary 1. For any two party function $f$ that can be computed using a boolean circuit of size $s$, there is a UC-secure protocol for $\mathcal{F}_{f}$ in the $\mathcal{F}_{\mathrm{BSC}}$-hybrid model, with $O(s)+\operatorname{poly}(k)$ bits of communication.

\subsection{A Constant-Rate String-OT Protocol}

We denote by $\mathcal{F}_{\text {string-OT }[\ell]}$ a string-OT functionality for which the sender's inputs are two strings from $\{0,1\}^{\ell}$. In this section we prove the following.

Lemma 1. There exists a protocol which securely realizes a single instance of

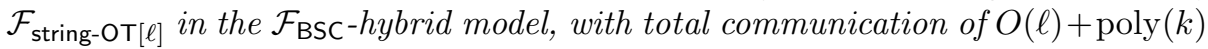
bits.

This constant-rate protocol for $\mathcal{F}_{\text {string-От in the }} \mathcal{F}_{\text {BSC-hybrid model is constructed }}$ in three steps. The construction relies on an intermediate functionality, namely $\mathcal{F}_{\text {OLE }}$ (or more precisely, $\widetilde{\mathcal{F}}_{\text {OLE }}$ ). The $\mathcal{F}_{\text {OLE }}$ functionality (OLE stands for Oblivious Linear function Evaluation) over the field $\mathbb{F}$ evaluates the following function: it takes $p, r \in \mathbb{F}$ from Alice and $q \in \mathbb{F}$ from Bob and outputs $p q+r$ to Bob (and sends an empty output to Alice). $\widetilde{\mathcal{F}}_{\text {OLE }}$ is the error-prone version of $\mathcal{F}_{\text {OLE }}$ as defined in Section 2, For simplicity we omit here the error parameter, which will be chosen as a sufficiently small constant.

Our protocol for $\mathcal{F}_{\text {string-OT }}$ in the $\mathcal{F}_{\mathrm{BSC}}$-hybrid model is constructed by composing the following protocols:

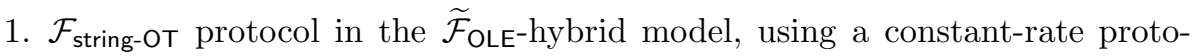
col that relies on a constant-rate arithmetic encoding scheme as defined in Section 2 . 


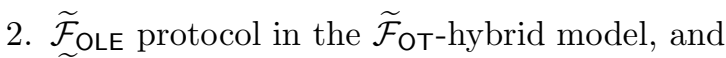

3. $\widetilde{\mathcal{F}}_{\text {OT }}$ protocol in the $\mathcal{F}_{\mathrm{BSC}}$-hybrid model.

The second step is obtained by applying Theorem 1 to any OT-based protocol for $\mathcal{F}_{\text {OLE }}$ (e.g., [26 24] ), where the latter is invoked with a sufficiently large (but constant) security parameter. The third step is obtained by similarly applying Theorem 1 to any protocol for $\mathcal{F}_{\text {OT }}$ from $\mathcal{F}_{\text {BSC }}$ (e.g., 10]). See full version for further details on the last two steps. In the rest of this section we focus on the first step.

Reducing $\mathcal{F}_{\text {string-OT }}$ to $\widetilde{\mathcal{F}}_{\text {OLE }}$. This construction uses an arithmetic encoding scheme as defined in Section 2, with parameters $0<\rho, \delta, \delta^{\prime}<1$ and a constantsize $\mathbb{F}$. We point out that a given arithmetic encoding scheme can be considered to be a scheme with any smaller (positive) value of $\delta$ than originally specified. Hence, to suit the requirements of our protocol, we shall assume that $\delta<\left(1-\delta^{\prime}\right) \rho / 6$. The protocol is in the $\tilde{\mathcal{F}}_{\text {OLE }}^{(\phi)}$-hybrid where $\phi \leq \delta / 2$.

We shall also use a strong randomness extractor Ext in our construction - a family of pairwise independent hash functions suffices.

Suppose Alice's inputs are two strings $s_{0}$ and $s_{1}$ and Bob's input is a choice

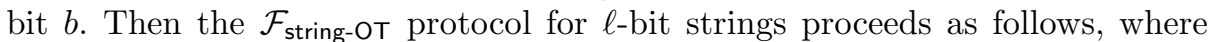
Encode and Encode ${ }^{\prime}$ map strings in $\mathbb{F}^{m}$ to strings in $\mathbb{F}^{n}$ and $d=\lfloor\delta n\rfloor$. The parameter $m$ (and the parameters of the extractor Ext) will be chosen such that for a string $x$ distributed uniformly over any set of size $|\mathbb{F}|^{m\left(1-\delta^{\prime}\right) / 2}$ or more, then $\operatorname{Ext}(x ; h) \in\{0,1\}^{\ell}$ (where $\ell=\Omega(m)$ is the length of Alice's strings), and $(h, \operatorname{Ext}(x ; h)$ ) (for a randomly chosen $h$ ) is almost uniformly random (up to a statistical distance of $\left.2^{-\Omega(k)}\right)$ over its range, even given up to $3 d \log |\mathbb{F}|+\ell$ additional bits of information about $x$.

- Alice's input is $\left(s_{0}, s_{1}\right)$ where $s_{0}, s_{1} \in\{0,1\}^{\ell}$, and Bob's input is $b \in\{0,1\}$.

- Alice lets $X_{0}=\operatorname{Encode}\left(x_{0}\right)$, and $X_{1}=\operatorname{Encode}\left(x_{1}\right)$, where $x_{0}, x_{1}$ are randomly drawn from $\mathbb{F}^{m}$. She also sets $Z=\operatorname{Encode}^{\prime}\left(0^{m}\right)$.

- Bob lets $B=\operatorname{Encode}\left(b^{m}\right)$ (the bit $b$ is identified with 0 or 1 in $\mathbb{F}$ ).

- They invoke $n$ instances of the $\tilde{\mathcal{F}}_{\text {OLE }}^{(\phi)}$ functionality, as follows. For each $i \in[n]$, Alice inputs $\left(p_{i}, r_{i}\right)=\left(X_{1}^{(i)}-X_{0}^{(i)}, X_{0}^{(i)}+Z^{(i)}\right)$ and Bob inputs $q_{i}=B^{(i)}$ to an instance of $\widetilde{\mathcal{F}}_{\mathrm{OLE}}^{(\phi)}$, and Bob gets the output $y_{i}=p_{i} q_{i}+r_{i} .\left(X^{(i)}\right.$ stands for the $i^{\text {th }}$ bit of the vector $X$.) The vector $y \in \mathbb{F}^{n}$ that Bob gets from this is a (possibly) noisy version of $X_{0} *(1-B)+X_{1} * B+Z$, which in turn is in the support of Encode' $\left(x_{b}\right)$. Bob sets $x_{b}=\operatorname{Decode}^{\prime}(y)$.

- Alice picks two seeds $h_{0}, h_{1}$ for Ext and lets $w_{0}=s_{0} \oplus \operatorname{Ext}\left(x_{0} ; h_{0}\right)$ and $w_{1}=s_{1} \oplus \operatorname{Ext}\left(x_{1} ; h_{1}\right)$. (The parameters of Ext are chosen as mentioned above.) She sends $\left(h_{0}, h_{1}, w_{0}, w_{1}\right)$ to Bob.

- Bob obtains $s_{b}=w_{b} \oplus \operatorname{Ext}\left(x_{b} ; h_{b}\right)$.

It is easy to see that the above protocol has a constant rate (since $\ell=\Omega(m)$ ). To prove the UC security of the protocol, we need to consider the case where both parties are honest, as well as when one of the parties is corrupt. In all cases, 
note that at most $2 \phi n<d$ out of $n$ instances of $\widetilde{\mathcal{F}}_{\text {OLE }}^{(\phi)}$ will let the adversary control them, except with negligible probability. In the simulation for all three cases, the simulator starts off by faithfully simulating whether each instance of $\widetilde{\mathcal{F}}_{\mathrm{OLE}}^{(\phi)}$ lets the adversary control it or not. If more than $d$ instances yield to adversarial control, the simulation aborts; as in the real execution, this happens with negligible probability. In the rest of the analysis, we condition on this not happening in the real execution as well as in the simulation.

When neither party is corrupted, security follows from the error-correcting property of Decode'. See full version for details.

Security When Neither Party Is Corrupt. In the simulation (i.e., ideal execution of $\left.\mathcal{F}_{\text {string-OT }}\right)$, Bob's output is always $s_{b}$ when Alice's inputs are $\left(s_{0}, s_{1}\right)$ and Bob's input is $b$. In the real execution of the protocol (conditioned on less than $d$ instances of $\widetilde{\mathcal{F}}_{\text {OLE }}^{(\phi)}$ being under adversarial control) the vector $y$ received by Bob has a Hamming distance of less than $d$ from a vector in the support of Encode $^{\prime}\left(x_{b}\right)$. So, by the error-correcting guarantee of Decode' Bob recovers $x_{b}$, and hence outputs $s_{b}$ correctly.

Security against Corrupt Alice. Here the simulation proceeds in two steps. First, Alice's view is completely straight-line simulated (if well-formed messages are not received from Alice in any round, then Bob aborting the protocol can be simulated). Next Bob's view is sampled for the two cases $b=0$ and $b=1$, conditioned on Alice's view, from which Bob's outputs for each case, denoted $s_{0}$ and $s_{1}$, respectively, are obtained. To complete the simulation the simulator sends $\left(s_{0}, s_{1}\right)$ as the input to the ideal $\mathcal{F}_{\text {string-От functionality. Details of the two }}$ steps follow.

Conditioned on $\widetilde{\mathcal{F}}_{\text {OLE }}^{(\phi)}$ yielding to the adversary at most $d$ times, Alice sees at most $d$ entries of the encoding of $B$ and this can be perfectly simulated since they are independent of Bob's input. So first the simulator will sample the (at most) $d$ entries for $B$ and hands them over to the Alice as the message from the instances of $\widetilde{\mathcal{F}}_{\text {OLE }}^{(\phi)}$ controlled by her. Then it receives from Alice the output for Bob from these instances. Further, the simulator receives all but $d$ entries of $\left(X_{0}, X_{1}, Z\right)$ from Alice as inputs to the instances of $\tilde{\mathcal{F}}_{\text {OLE }}^{(\phi)}$ not controlled by her. In the next round, the simulator receives $\left(h_{0}, h_{1}, w_{0}, w_{1}\right)$ from Alice. This completes the first part of the simulation.

For the next part, the simulator picks $B_{0}=\operatorname{Encode}\left(0^{m}\right)$ and $B_{1}=\operatorname{Encode}\left(1^{m}\right)$ randomly, conditioned to match the $d$ coordinates of $B$ that were already simulated. Then, it computes $s_{0}$ as what Bob would compute in the protocol if it uses $B=B_{0}$ and receives the messages implied by what Alice sent to the simulator in the first part. Similarly, it computes $s_{1}$ if Bob used $B=B_{1}$. Then the simulator will send $\left(s_{0}, s_{1}\right)$ to the functionality.

Given our conditioning the real and simulated executions on there being no more than $d$ instances of $\widetilde{\mathcal{F}}_{\text {OLE }}^{(\phi)}$ under adversarial control, this is a perfect simulation. Thus over all, this gives a statistically good simulation. 
Security against Corrupt Bob. If Bob is corrupt, then he may not input a valid $B$ in the range of $\operatorname{Encode}\left(0^{m}\right)$ or $\operatorname{Encode}\left(1^{m}\right)$. Nevertheless, we shall see that by using appropriate encodings and the extractor, there is a string $s_{b}$ such that Bob learns a negligible amount of information about $s_{1-b}$.

Note that what Bob learns by Step 2 of the protocol is given by a system of $n$ linear equations (defined by his input $B$ to $\widetilde{\mathcal{F}}_{\text {OLE }}$ ) over $x_{0}, x_{1}$ and the random elements used by Alice in forming the encodings $X_{0}, X_{1}$ and the entries of $Z$, and the values of $X_{0}, X_{1}$ and $Z$ at no more than $d$ randomly chosen entries. Alice's secrets at this point are two vectors $x_{0}, x_{1}$ of length only $m$, so it is non-trivial to ensure that the information that Bob learns (which is more than $n$ field elements, and typically $n>2 m$ ) does not contain both $x_{0}$ and $x_{1}$. This is ensured by the blinding: intuitively, $Z$ encodes at least $t^{\prime}:=n-m\left(1+\delta^{\prime}\right)$ random field elements, and so effectively Bob learns at most as much information about $\left(x_{0}, x_{1}\right)$ as from $n-t^{\prime}=m\left(1+\delta^{\prime}\right)<2 m$ linear equations.

More formally, let $U$ denote the output vector in $\mathbb{F}^{n}$ obtained by Bob from $\widetilde{\mathcal{F}}_{\text {OLE }}$, and let $\widetilde{U}$ denote the (at most $3 d$ ) field elements learned by Bob from corrupted instances of $\widetilde{\mathcal{F}}_{\text {OLE }}$. It can be shown (see full version) that for any possible value of Bob's $\widetilde{\mathcal{F}}_{\text {OLE }}$ input $B$ there is $c \in\{0,1\}$ such that the distribution of $x_{c}$ conditioned on $B$ and any possible $U$ is uniform in an affine space whose dimension is at least $\frac{m\left(1-\delta^{\prime}\right)}{2}$.

Note that $c$ as above can be efficiently computed by solving for $x_{0}$ and $x_{1}$ from the equations defined by $B$ and Encode', and checking the dimension of their solution spaces. The simulator first perfectly simulates $B, \widetilde{U}$, then uses $B$ to compute $c$, and then sends $b=1-c$ to the functionality to obtain $s_{b}$. To complete the simulation, the simulator sets $s_{c}=0$ and generates Alice's messages (to both Bob and $\widetilde{\mathcal{F}}_{\text {OLE }}$ ) at random conditioned on the values of $B, \widetilde{U}$ that were already simulated. The correctness of the simulator follows from the fact that in the real protocol, conditioned on the choice of $B$, the string $\operatorname{Ext}\left(x_{c} ; h_{c}\right)$ masking $s_{c}$ is almost uniformly random even when further conditioned on the remaining view of Bob. This follows from the fact that $\widetilde{U}$ and $\left(h_{b}, \operatorname{Ext}\left(x_{b} ; h_{b}\right)\right)$ leak at most $3 d \log |\mathbb{F}|$ and $\ell$ additional bits of information, respectively, which our choice of parameters for Ext tolerates. See full version for further details.

\subsection{Error-Tolerant Protocol for $\mathcal{F}_{\mathrm{OT}}$ over $\mathcal{F}_{\mathrm{BSC}}$}

Error-tolerance. We say that a protocol $\pi$ is an error-tolerant protocol for $\mathcal{F}$ in the $\mathcal{G}$-hybrid model if it is secure against adaptive passive corruption, and in addition tolerates active corruption of a small constant fraction of $\mathcal{G}$ instances that it invokes. More formally, we can define a modified functionality $\mathcal{G}^{\prime}$, which behaves exactly as $\mathcal{G}$ until a new command corrupt is received as input from the adversary; if this command is received, then this instance of $\mathcal{G}$ will yield to adversarial control - i.e., send its current view to the adversary, forward immediately any subsequent message that it receives, and send messages to other parties in the protocol as instructed by the adversary. $\pi$ is called a $\epsilon_{0}$-errortolerant protocol for $\mathcal{F}$ in the $\mathcal{G}$-hybrid model if $\pi$ is a secure protocol for $\mathcal{F}$ in the $\mathcal{G}^{\prime}$-hybrid model against adaptive passive corruption, against the class of 
adversaries who send out the corrupt command to at most $\epsilon_{0} T$ of $\mathcal{G}^{\prime}$ instances, where $T$ is (an upperbound on) the total number of $\mathcal{G}$ instances invoked by $\pi$. We will call $\pi$ simply an error-tolerant protocol if it is $\epsilon_{0}$-error-tolerant for any constant $\epsilon_{0}>0$.

As described above in the inner protocol in our construction, we will require a constant-rate error-tolerant protocol for $\mathcal{F}_{\text {OT }}$ in the $\mathcal{F}_{\text {BSC }}$-hybrid model.

We observe that such a protocol is implicit in a result in [20. They present a constant-rate $\mathrm{OT}$ protocol in the $\mathcal{F}_{\mathrm{BSC}}$-hybrid model which is secure against adaptive passive adversaries. This construction starts with a simple passivesecure constant-rate protocol $\Phi$ for $\mathcal{F}_{\mathrm{OT}}$ in the $\mathcal{F}_{\mathrm{BSC}}$-hybrid model, with a small but constant probability of error, and then uses a constant-rate combiner to reduce the error to negligible. This combiner uses each candidate $\mathcal{F}_{\text {OT }}$ instance once, and (passive-securely) realizes a constant fraction of $\mathcal{F}_{\text {OT }}$ instances. As mentioned in [20], the "error-tolerant" version of their combiner allows a small fraction of the candidate $\mathcal{F}_{\text {OT }}$ instances to be actively and adaptively corrupted, though requires the parties themselves to follow the combiner's protocol honestly. The combiner corresponds to a constant-rate protocol for $\mathcal{F}_{\text {OT }}$ in the $\mathcal{F}_{\text {OT-hybrid }}$ model with error tolerance as we have defined above. By composing this protocol with $\Phi$, we get a constant-rate protocol for $\mathcal{F}_{\text {OT }}$ in the $\mathcal{F}_{\text {BSC-hybrid model, }}$ with the property that if a small constant fraction of the instances of $\mathcal{F}_{\mathrm{BSC}}$ are corrupted (resulting in the corruption of a small fraction of $\mathcal{F}_{\text {OT }}$ instances used by the combiner protocol), security remains intact.

Lemma 2. [20] There is a constant-rate, error-tolerant protocol for $\mathcal{F}_{\mathrm{OT}}$ in the $\mathcal{F}_{\mathrm{BSC}}$-hybrid model.

\subsection{An Extension to the IPS Compiler}

IPS compiler requires a semi-honest inner protocol over $\mathcal{F}_{\text {OT }}$. We need to extend this compiler to work with inner protocols over $\mathcal{F}_{\text {BSC }}$. The IPS compiler depends on being able to monitor the use of $\mathcal{F}_{\text {OT }}$ channels with a good probability of catching errors; however, one cannot monitor the $\mathcal{F}_{\mathrm{BSC}}$ functionality at the same level. Hence we shall depend on the slightly stronger error-tolerance guarantee of the inner protocol. Here we shall limit ourselves to the 2-party setting (since we are interested in a 2-party functionality, namely $\mathcal{F}_{\mathrm{OT}}$ ).

Below we state the extension of the IPS compiler (with the new elements underlined). See full version for a proof.

Lemma 3. Suppose $\Pi$ is a protocol among $n=\Theta(k)$ servers and 2 clients, for a 2-party functionality $\mathcal{F}$ between the clients, with $U C$-security against adaptive, active corruption of $\Omega(n)$ servers and adaptive, active corruption of (any number of) clients. Suppose $\rho^{\mathcal{F}_{\mathrm{BSC}}}$ is a 2-party protocol in the $\mathcal{F}_{\mathrm{BSC}}$-hybrid model, that realizes the functionality of each server in the protocol $\overline{\Pi \text {, with error tolerance. }}$. Then there is a 2-party protocol (compiled protocol) for the functionality $\mathcal{F}$ in the $\left(\mathcal{F}_{\mathrm{BSC}}, \mathcal{F}_{\text {string-OT }}\right)$-hybrid model, with $U C$-security against adaptive, active 
adversaries. Further, if the (insecure) protocol obtained by directly implementing each server of $\Pi$ using $\rho^{\mathcal{F}_{\mathrm{BSC}}}$ has constant rate, then the compiled protocol has constant rate too.

Putting Things Together. The final protocol is obtained from Lemma 3 by using the following outer and inner protocols. The outer protocol is the one used in Section 5.1 of 24] (based on [138) applied to the functionality which realizes $n$ instances of OT. The inner protocol is the standard OT-based implementation of the GMW protocol in the semi-honest OT-hybrid model [18], except that the OT instances consumed by this protocol are implemented using the error-tolerant protocol of Lemma 2, The watchlists are implemented using the protocol of Lemma1.

\section{References}

1. Ahlswede, R., Csiszar, I.: On Oblivious Transfer Capacity. In: ISIT 2007, pp. 20612064 (2007)

2. Bennett, C.H., Brassard, G., Crépeau, C., Maurer, U.: Generalized privacy amplification. IEEE Transactions on Information Theory 41, 1915-1923 (1995)

3. Bennett, C.H., Brassard, G., Robert, J.-M.: Privacy Amplification by Public Discussion. SIAM J. Comput. 17(2), 210-229 (1988)

4. Brassard, G., Crépeau, C., Robert, J.-M.: All-or-nothing disclosure of secrets. In: Odlyzko, A.M. (ed.) CRYPTO 1986. LNCS, vol. 263, pp. 234-238. Springer, Heidelberg (1987)

5. Canetti, R.: Security and composition of multiparty cryptographic protocols. Journal of Cryptology 13(1), 143-202 (2000)

6. Canetti, R.: Universally Composable Security: A New Paradigm for Cryptographic Protocols. In: FOCS 2001, pp. 136-145 (2001)

7. Cascudo, I., Cramer, R., Xing, C.: The Torsion-Limit for Algebraic Function Fields and Its Application to Arithmetic Secret Sharing. In: Crypto 2011 (2011)

8. Chen, H., Cramer, R.: Algebraic geometric secret sharing schemes and secure multiparty computations over small fields. In: Dwork, C. (ed.) CRYPTO 2006. LNCS, vol. 4117, pp. 521-536. Springer, Heidelberg (2006)

9. Crépeau, C.: Efficient cryptographic protocols based on noisy channels. In: EUROCRYPT 1997, pp. 306-317 (1997)

10. Crépeau, C., Kilian, J.: Achieving oblivious transfer using weakened security assumptions. In: FOCS 1988, pp. 42-52 (1988)

11. Crépeau, C., Morozov, K., Wolf, S.: Efficient Unconditional Oblivious Transfer from Almost Any Noisy Channel. In: Blundo, C., Cimato, S. (eds.) SCN 2004. LNCS, vol. 3352, pp. 47-59. Springer, Heidelberg (2005)

12. Damgård, I., Fehr, S., Morozov, K., Salvail, L.: Unfair Noisy Channels and Oblivious Transfer. In: Naor, M. (ed.) TCC 2004. LNCS, vol. 2951, pp. 355-373. Springer, Heidelberg (2004)

13. Damgård, I., Ishai, Y.: Scalable Secure Multiparty Computation. In: Dwork, C. (ed.) CRYPTO 2006. LNCS, vol. 4117, pp. 501-520. Springer, Heidelberg (2006)

14. Damgård, I., Kilian, J., Salvail, L.: On the (Im)possibility of Basing Oblivious Transfer and Bit Commitment on Weakened Security Assumptions. In: Stern, J. (ed.) EUROCRYPT 1999. LNCS, vol. 1592, pp. 56-73. Springer, Heidelberg (1999)

15. Even, S., Goldreich, O., Lempel, A.: A randomized protocol for signing contracts. Communications of the ACM 28(6), 637-647 (1985)

16. Garcia, A., Stichtenoth, H.: On the asymptotic behavior of some towers of function fields over finite fields. Journal of Number Theory 61(2), 248-273 (1996) 
17. Gemmell, P., Sudan, M.: Highly Resilient Correctors for Polynomials. Information Processing Letters 43(4), 169-174 (1992)

18. Goldreich, O.: Foundations of Cryptography, vol. 2. Cambridge University Press, Cambridge (2004)

19. Goldreich, O., Micali, S., Wigderson, A.: How to play any mental game. In: STOC 1987, pp. 218-229 (1987)

20. Harnik, D., Ishai, Y., Kushilevitz, E., Nielsen, J.B.: OT-Combiners via Secure Computation. In: Canetti, R. (ed.) TCC 2008. LNCS, vol. 4948, pp. 393-411. Springer, Heidelberg (2008)

21. Harnik, D., Kilian, J., Naor, M., Reingold, O., Rosen, A.: On tolerant combiners for oblivious transfer and other primitives. In: EUROCRYPT 2005, pp. 96-113 (2005)

22. Imai, H., Morozov, K., Nascimento, A.: Efficient Oblivious Transfer Protocols Achieving a Non-Zero Rate from Any Non-Trivial Noisy Correlation. In: Desmedt, Y. (ed.) ICITS 2007. LNCS, vol. 4883, pp. 183-194. Springer, Heidelberg (2009)

23. Ishai, Y., Kushilevitz, E., Ostrovsky, R., Sahai, A.: Zero-knowledge from secure multiparty computation. In: STOC 2007, pp. 21-30 (2007)

24. Ishai, Y., Prabhakaran, M., Sahai, A.: Founding Cryptography on Oblivious Transfer - Efficiently. In: Wagner, D. (ed.) CRYPTO 2008. LNCS, vol. 5157, pp. 572-591. Springer, Heidelberg (2008)

25. Ishai, Y., Kushilevitz, E., Ostrovsky, R., Sahai, A.: Extracting Correlations. In: FOCS 2009, pp. 261-270 (2009)

26. Kilian, J.: Founding cryptography on oblivious transfer. In: STOC 1988, pp. 20-31 (1988)

27. Kilian, J.: More general completeness theorems for secure two-party computation. In: STOC 2000, pp. 316-324 (2000)

28. Maurer, U.: Perfect Cryptographic Security from Partially Independent Channels. In: STOC 1991, pp. 561-571 (1991)

29. Maurer, U.M., Pietrzak, K., Renner, R.: Indistinguishability Amplification. In: Menezes, A. (ed.) CRYPTO 2007. LNCS, vol. 4622, pp. 130-149. Springer, Heidelberg (2007)

30. Nascimento, A., Winter, A.: On the Oblivious Transfer Capacity of Noisy Correlations. In: ISIT 2006, pp. 1871-1875 (2006)

31. Przydatek, B., Wullschleger, J.: Error-Tolerant Combiners for Oblivious Primitives. In: Aceto, L., Damgård, I., Goldberg, L.A., Halldórsson, M.M., Ingólfsdóttir, A., Walukiewicz, I. (eds.) ICALP 2008, Part II. LNCS, vol. 5126, pp. 461-472. Springer, Heidelberg (2008)

32. Rabin, M.O.: How to exchange secrets by oblivious transfer. In: TR 1981, Harvard (1981)

33. Shannon, C.E.: A mathematical theory of communication. Bell System Technical Journal 27, 379-423, 623-656 (1948)

34. Wiesner, S.: Conjugate coding. SIGACT News 15(1), 78-88 (1983)

35. Winkler, S., Wullschleger, J.: On the Efficiency of Classical and Quantum Oblivious Transfer Reductions. In: Rabin, T. (ed.) CRYPTO 2010. LNCS, vol. 6223, pp. $707-$ 723. Springer, Heidelberg (2010)

36. Winter, A., Nascimento, A.C.A., Imai, H.: Commitment Capacity of Discrete Memoryless Channels. In: IMA Int. Conf. pp. 35-51 (2003)

37. Wullschleger, J.: Oblivious Transfer from Weak Noisy Channels. In: Reingold, O. (ed.) TCC 2009. LNCS, vol. 5444, pp. 332-349. Springer, Heidelberg (2009)

38. Wyner, A.D.: The wire-tap channel. Bell Cyst. Tech. J. 54, 1355-1387 (1975)

39. Yao, A.C.: How to generate and exchange secrets. In: FOCS 1986, pp. 162-167 (1986) 


\section{A Arithmetic Encoding from MPC-Friendly Codes}

In this section, we briefly sketch how an implementation of our notion of an arithmetic encoding scheme (Encode, Encode', Decode') (as defined in Section 2) follows from the literature.

Below we recall (verbatim) the notion of MPC-friendly codes from [25], which almost have all the properties we need. (The parameter $k$ in this section should not be confused with the use of $k$ as a security parameter in the rest of the paper.)

Claim ([25], implicit in [8]). There exists a finite field $\mathbb{F}$ and an efficiently constructible family of linear error-correcting codes $C_{k}: \mathbb{F}^{k} \rightarrow \mathbb{F}^{n_{k}}$ with the following properties: (1) $n_{k}=O(k)$; (2) The dual distance of $C_{k}$ is $\delta_{k}=\Omega(k)$; (3) The linear code $C_{k}^{\prime}$ spanned by all entrywise-products of pairs of codewords in $C_{k}$ supports efficient decoding of up to $\mu_{k}=\Omega(k)$ errors. (The entrywise product of $\left(c_{1}, \ldots, c_{n}\right)$ and $\left(c_{1}^{\prime}, \ldots, c_{n}^{\prime}\right)$ is $\left(c_{1} c_{1}^{\prime}, \ldots, c_{n} c_{n}^{\prime}\right)$.)

A family of codes $C_{k}$ as above can be obtained from the construction of Garcia and Stichtenoth [16. An efficient decoder for $C_{k}^{\prime}$ can be obtained via the Gemmel-Sudan generalization of the Welch-Berlekamp decoder for Reed-Solomon codes 17.

The one stronger property that we need here (beyond what was needed in [25]), in order to guarantee that Encode' generates the amount of entropy that we need, is a "near-MDS" property of the code $C_{k}^{\prime}$. Specifically, it suffices to ensure that, for a small enough constant $\delta_{0}>0$, we have:

$$
\left(n_{k}-\operatorname{dim}\left(C_{k}^{\prime}\right)-\Delta_{k}\right)<\delta_{0} k
$$

Indeed, this follows immediately from the construction of [16], which in fact allows us to obtain $\delta_{0}=o(1)$.

The code $C_{k}$ corresponds to the Encode algorithm, and the code $C_{k}^{\prime}$ corresponds to the Encode' algorithm. In both cases, not just the message, but additional randomness would also be encoded (in the standard method for secret sharing). More specifically, it will suffice to have $\operatorname{Encode}(x)$ (respectively, Encode $\left.^{\prime}(x)\right)$ sample a random codeword $y$ in the range of $C_{k}$ (respectively, $C_{k}^{\prime}$ ) such that $y$ has $x$ as a prefix, and set the encoding to be $y$ modified by dropping this prefix. This can be done efficiently since the codes are linear (by solving a system of linear equations over $x$ and randomly chosen field elements). In particular, if $C_{k}$ and $C_{k}^{\prime}$ are systematic codes, then Encode $(x)$ (respectively, Encode $(x)$ ) will pick a random vector $r$ of the appropriate dimension, let $y=C_{k}(x \| r)$ (respectively, $y=C_{k}^{\prime}(x \| r)$ ), and output the last $n-m$ entries of $y$.

It is instructive to note that Reed-Solomon codes satisfy all the properties we need, except that Reed-Solomon codes would require the size of the field $\mathbb{F}$ to grow linearly with $k$. One could use Reed-Solomon codes in our constructions (instead of algebraic geometric codes) at the cost of a polylogarithmic deterioration of the parameters. 